\title{
A new algorithm for calculating the curvature perturbations in stochastic inflation
}

\author{
Tomohiro Fujita, ${ }^{a, b}$ Masahiro Kawasaki, ${ }^{a, c}$ Yuichiro Tada ${ }^{a, b}$ and \\ Tomohiro Takesako ${ }^{c}$
}

\author{
${ }^{a}$ Kavli Institute for the Physics and Mathematics of the Universe (Kavli IPMU), TODIAS, \\ the University of Tokyo, 5-1-5 Kashiwanoha, Kashiwa, 277-8583, Japan \\ ${ }^{b}$ Department of Physics, the University of Tokyo, Bunkyo-ku 113-0033, Japan \\ ${ }^{c}$ Institute for Cosmic Ray Research, the University of Tokyo, 5-1-5 Kashiwa-no-Ha, Kashiwa, \\ Chiba, 277-8582, Japan \\ ${ }^{d}$ Advanced Leading Graduate Course for Photon Science (ALPS), the University of Tokyo, \\ Bunkyo-ku 113-0033, Japan \\ E-mail: tomohiro.fujita@ipmu.jp, kawasaki@icrr.u-tokyo.ac.jp, \\ yuichiro.tada@ipmu.jp, takesako@icrr.u-tokyo.ac.jp
}

\begin{abstract}
We propose a new approach for calculating the curvature perturbations produced during inflation in the stochastic formalism. In our formalism, the fluctuations of the efoldings are directly calculated without perturbatively expanding the inflaton field and they are connected to the curvature perturbations by the $\delta N$ formalism. The result automatically includes the contributions of the higher order perturbations because we solve the equation of motion non-perturbatively. In this paper, we analytically prove that our result (the power spectrum and the nonlinearity parameter) is consistent with the standard result in single field slow-roll inflation. We also describe the algorithm for numerical calculations of the curvature perturbations in more general inflation models.
\end{abstract}

Keywords: cosmological perturbation theory, inflation, physics of the early universe

ArXiv ePrint: 1308.4754 


\section{Contents}

1 Introduction 1

2 Stochastic inflation $\quad 2$

3 Analytic calculation of curvature perturbations 4

3.1 First passage time 5

$\begin{array}{lll}3.2 & \text { Power spectrum } & 6\end{array}$

3.3 Non-Gaussianity 8

4 Extension to more general case $\quad 10$

5 Conclusion $\quad 12$

\section{Introduction}

Inflation [1-5] is a plausible model of the early universe and now believed by many physicists. It solves various problems such as the horizon problem, the flatness problem, the monopole problem and so on [6-8]. Furthermore, simple inflation models produce almost scale invariant curvature perturbations which are verified by the observations of the cosmic microwave background $(\mathrm{CMB})$ or the large scale structure [9].

Inflation is presumably caused by one or more real scalar fields which are called the inflatons and the curvature perturbations are originated from the quantum fluctuations of these scalar fields. In usual analysis of the dynamics of inflation, the inflaton field is divided into the spatially homogeneous classical background part and the perturbative quantum fluctuations part. Then the contributions of the quantum fluctuations to the background dynamics are often neglected. However, in some inflation models, the quantum kicks to the background are essential. For example, it is possible that the background inflaton field not only rolls down but also climbs up the potential due to the quantum kicks. In these cases, inflation will not end eternally (which is called eternal inflation $[10,11]$ ). The stochastic formalism [12-24] can take into account the quantum effects by treating the quantum kicks as stochastic noise in the equation of motion as we will see in section 2 .

When the quantum kicks are taken into consideration, the background field is no longer homogeneous because the noise in each Hubble scale region has independent values. In other words, each Hubble scale universe evolves separately. Therefore a linear perturbation theory where the background field is assumed to be homogeneous might not give correct curvature perturbations. Especially in a highly non-Gaussian case, for example, it is difficult to calculate the curvature perturbations in a perturbative theory because the contributions of the higher order perturbations are crucial.

In this paper, we propose a new algorithm for calculating the curvature perturbations in the stochastic formalism. In our algorithm, the fluctuations of the e-foldings are directly calculated and they are connected to the curvature perturbations with use of the $\delta N$ formalism [25-29]. Since we do not need to expand the inflaton field perturbatively in our formalism, results automatically include the contributions of the higher order perturbations 
of the inflaton field. Therefore, when these contributions are important, such as highly nonGaussian inflation models, it becomes a quite useful method. It is also advantageous when we calculate the higher order quantities such as the non-Gaussianity of the curvature perturbations. According to the recent observation of CMB [9], the curvature perturbations are so small and almost Gaussian at the CMB scale, but it is possible that they are large and/or quite non-Gaussian at smaller scales, which leads to significant cosmological effect. In fact, some interesting astronomical objects like primordial black holes (PBHs) [39-42] and ultracompact minihalos (UCMHs) [43-45] can be formed when the curvature perturbations are large and non-Gaussian. Our non-perturbative formalism is needed in such cases.

If we consider a single field inflation model with sufficiently flat inflaton potential, the curvature perturbations can be analytically calculated even in our formalism with the help of techniques of stochastic calculus. Then we can compare the power spectrum and the nonlinearity parameter of the curvature perturbations calculated in our formalism with those in usual perturbation theory. It is found the results are consistent and thus our formalism is verified at least in single slow-roll inflation models. Moreover, our formalism is applicable to wider class of inflation models by using numerical calculations. We will describe a calculation algorithm for more general inflation models.

The power spectrum of the curvature perturbations was firstly derived in the stochastic formalism by Kunze [32]. Ref. [32] obtained the power spectrum from the time derivative of the variance of the e-foldings, which we also adopt in our formalism. However, in that article, the inflaton field is expanded perturbatively with respect to the noise term $[33,34]$ and the validity of this perturbative expansion is non-trivial. Furthermore, their result deviates from the standard result of the usual perturbation theory even in single slow-roll inflation where the standard linear perturbation theory works well and gives precise results. Therefore, the interpretation of this deviation is difficult. In contrast, in our formalism, the curvature perturbations can be calculated non-perturbatively and the result is consistent with the standard formalism. ${ }^{1}$

The structure of this paper as follows. In section 2, we briefly review the stochastic formalism. In section 3, we explain the techniques of stochastic calculus in the first place. Then we will show how the power spectrum and the non-Gaussianity of the curvature perturbations can be calculated and prove that the result in single slow-roll inflation is consistent with that of the standard formalism. In section 4, the extension to more general inflation models is discussed. Finally, section 5 is devoted to conclusions of this paper.

\section{Stochastic inflation}

In the stochastic approach, we divide the real inflaton field $\phi$ into two parts, IR mode and UV mode, as

$$
\begin{aligned}
\phi(\mathbf{x}, t) & =\phi_{\mathrm{IR}}(\mathbf{x}, t)+\phi_{\mathrm{UV}}(\mathbf{x}, t), \\
\phi_{\mathrm{IR}}(\mathbf{x}, t) & =\int \frac{d^{3} k}{(2 \pi)^{3}} \theta(\epsilon a(t) H(t)-k) \phi_{\mathbf{k}}(t) e^{-i \mathbf{k} \cdot \mathbf{x}}, \\
\phi_{\mathrm{UV}}(\mathbf{x}, t) & =\int \frac{d^{3} k}{(2 \pi)^{3}}(1-\theta(\epsilon a(t) H(t)-k)) \phi_{\mathbf{k}}(t) e^{-i \mathbf{k} \cdot \mathbf{x}},
\end{aligned}
$$

\footnotetext{
${ }^{1}$ During preparation of this paper, another approach to curvature perturbations in the stochastic formalism has been provided in ref. $[35,36]$ and they also seem to relate with our work.
} 
where $\phi_{\mathbf{k}}(t)$ is the Fourier mode of the inflaton field $\phi(\mathbf{x}, t), \theta(z)$ is the step function, $H(t)$ is the Hubble parameter, $a(t)$ is the scale factor and $\epsilon$ is a small constant parameter. In this paper, following the original works $[12,13]$, we choose the step function as the window function splitting the IR mode and the UV mode. ${ }^{2}$ We treat the IR mode as a classical stochastic background field and are interested only in this mode.

Let us consider the following action for the inflaton field:

$$
S=\int d^{4} x \sqrt{-g}\left(\frac{1}{2} \partial_{\mu} \phi \partial^{\mu} \phi-V(\phi)\right),
$$

and then the equations of motion in the Hamiltonian formulation are given by

$$
\left\{\begin{array}{l}
\pi=\dot{\phi} \\
\dot{\pi}+3 H \pi-a^{-2} \nabla^{2} \phi-V^{\prime}(\phi)=0
\end{array}\right.
$$

where a dot and a prime denote derivatives respect to the time and the inflaton field respectively. In a way similar to the inflaton field, we also divide the conjugate field $\pi$ into the IR and UV modes as

$$
\begin{aligned}
\pi_{\mathrm{IR}}(\mathbf{x}, t) & =\int \frac{d^{3} k}{(2 \pi)^{3}} \theta(\epsilon a(t) H(t)-k) \dot{\phi}_{\mathbf{k}}(t) e^{-i \mathbf{k} \cdot \mathbf{x}}, \\
\pi_{\mathrm{UV}}(\mathbf{x}, t) & =\int \frac{d^{3} k}{(2 \pi)^{3}}(1-\theta(\epsilon a(t) H(t)-k)) \dot{\phi}_{\mathbf{k}}(t) e^{-i \mathbf{k} \cdot \mathbf{x}} .
\end{aligned}
$$

It should be noted that $\dot{\phi}_{\mathrm{IR}} \neq \pi_{\mathrm{IR}}$ because of the time dependence of $\epsilon a H$. Substituting these definitions into the equations of motion (2.5) and (2.6), with approximation,

$$
V^{\prime}(\phi) \simeq V^{\prime}\left(\phi_{\mathrm{IR}}\right)+V^{\prime \prime}\left(\phi_{\mathrm{IR}}\right) \phi_{\mathrm{UV}},
$$

and equation of motion for $\phi_{\mathbf{k}}$,

$$
\ddot{\phi}_{\mathbf{k}}+3 H \dot{\phi}_{\mathbf{k}}+\left(\frac{k^{2}}{a^{2}}+V^{\prime \prime}\left(\phi_{\mathrm{IR}}\right)\right) \phi_{\mathbf{k}}=0
$$

we obtain the equations for the IR mode,

$$
\left\{\begin{array}{l}
\dot{\phi}_{\mathrm{IR}}=\pi_{\mathrm{IR}}+\epsilon a H^{2} \int \frac{d^{3} k}{\left(2 \pi^{3}\right)} \delta(\epsilon a H-k) \phi_{\mathbf{k}} e^{-i \mathbf{k} \cdot \mathbf{x}}, \\
\dot{\pi}_{\mathrm{IR}}=-3 H \pi_{\mathrm{IR}}-V^{\prime}\left(\phi_{\mathrm{IR}}\right)+\epsilon a H^{2} \int \frac{d^{3} k}{\left(2 \pi^{3}\right)} \delta(\epsilon a H-k) \pi_{\mathbf{k}} e^{-i \mathbf{k} \cdot \mathbf{x}} .
\end{array}\right.
$$

Here, the spatial derivative of the IR mode is neglected. Note that the Dirac delta function comes from the time derivative of the step function.

From now on, we consider only one spatial point. Then we can fix the spatial point to the origin, $\mathbf{x}=0$, without loss of generality. We denote the last terms of eqs. (2.11) and (2.12) as $\xi_{\phi}(t)$ and $\xi_{\pi}(t)$ respectively. Their expected values vanish because $\phi_{\mathbf{k}}$ and $\pi_{\mathbf{k}}$ are

\footnotetext{
${ }^{2}$ Note that a validity of a sharp cutoff window function is under discussion $[37,38]$.
} 
vacuum fluctuations. Furthermore, with $k_{c}(t)=\epsilon a H$, the expected value of $\xi_{\phi}(t) \xi_{\phi}\left(t^{\prime}\right)$ is given by

$$
\begin{aligned}
\left\langle\xi_{\phi}(t) \xi_{\phi}\left(t^{\prime}\right)\right\rangle & =k_{c}(t) k_{c}\left(t^{\prime}\right) H^{2} \int \frac{d^{3} k d^{3} k^{\prime}}{(2 \pi)^{6}} \delta\left(k_{c}(t)-k\right) \delta\left(k_{c}\left(t^{\prime}\right)-k^{\prime}\right)\left\langle\phi_{\mathbf{k}}(t) \phi_{\mathbf{k}^{\prime}}\left(t^{\prime}\right)\right\rangle \\
& =k_{c}^{2}(t) H^{2} \int \frac{d^{3} k}{(2 \pi)^{3}} \delta\left(k_{c}(t)-k\right) \frac{\delta\left(t-t^{\prime}\right)}{k_{c}(t) H} \frac{2 \pi^{2} \mathcal{P}_{\phi}(t, k)}{k^{3}} \\
& =H \mathcal{P}_{\phi}\left(t, k_{c}\right) \delta\left(t-t^{\prime}\right),
\end{aligned}
$$

where

$$
\mathcal{P}_{\phi}(t, k) \delta\left(\mathbf{k}+\mathbf{k}^{\prime}\right)=\frac{k^{3}}{2 \pi^{2}}\left\langle\phi_{\mathbf{k}} \phi_{\mathbf{k}^{\prime}}\right\rangle
$$

With $\epsilon \ll 1, k_{c}=\epsilon a H$ corresponds to the scale much larger than the horizon and we suppose that $\mathcal{P}_{\phi}\left(t, k_{c}\right) \rightarrow(H / 2 \pi)^{2}{ }^{3}$ Since $\xi_{\phi}(t)$ satisfies

$$
\left\langle\xi_{\phi}(t)\right\rangle=0, \quad\left\langle\xi_{\phi}(t) \xi_{\phi}\left(t^{\prime}\right)\right\rangle=\frac{H^{3}}{(2 \pi)^{2}} \delta\left(t-t^{\prime}\right),
$$

$\xi_{\phi}$ is white noise. By similar calculation, one can show the variance of $\xi_{\pi}$ vanishes. Thus $\xi_{\pi}$ can be neglected. Finally redefining $\xi=2 \pi \xi_{\phi} / H^{3 / 2}$, we obtain the following equations of motion:

$$
\left\{\begin{array}{l}
\dot{\phi}_{\mathrm{IR}}=\pi_{\mathrm{IR}}+\frac{H^{3 / 2}}{2 \pi} \xi, \\
\dot{\pi}_{\mathrm{IR}}=-3 H \pi_{\mathrm{IR}}-V^{\prime}\left(\phi_{\mathrm{IR}}\right), \\
\langle\xi(t)\rangle=0, \quad\left\langle\xi(t) \xi\left(t^{\prime}\right)\right\rangle=\delta\left(t-t^{\prime}\right),
\end{array}\right.
$$

Furthermore adopting the slow-roll approximation, $\dot{\pi}_{\mathrm{IR}} \simeq 0$, and using the Friedmann equation, $V \simeq 3 M_{p}^{2} H^{2}$, we obtain

$$
\dot{\phi}_{\mathrm{IR}}+2 M_{p}^{2} H^{\prime}=\frac{H^{3 / 2}}{2 \pi} \xi(t),
$$

where $M_{p} \approx 2.4 \times 10^{18} \mathrm{GeV}$ is the reduced planck mass. Therefore it is clear that the contributions of the quantum kicks to the coarse-grained field (i.e. IR mode) are represented as stochastic white noise.

\section{Analytic calculation of curvature perturbations}

In this section we develop the algorithm for calculating correlation functions of curvature perturbations of any order in the stochastic formalism. For this purpose, an analogy between slow-rolling inflaton dynamics and stochastic process is important. More specifically, a concept of the first passage time in stochastic calculus is crucial in order to use $\delta N$ formalism. Therefore, in the first place we briefly review stochastic calculus and then we explore the calculation of the curvature perturbation.

\footnotetext{
${ }^{3}$ This is a massless case spectrum. In standard single inflation, the inflaton is almost massless and this assumption is natural. However, in more general cases, the amplitude of the noise might not be constant. In those cases, numerical calculations are needed.
} 


\subsection{First passage time}

Dynamics of a slow-rolling inflaton in the stochastic formalism corresponds to a certain type of stochastic processes. As we see later, when the Hubble parameter is given by a linear function of $\phi$, the corresponding stochastic process is a Brownian motion with a constant drift. Thus, we describe it in this subsection. A Brownian motion with a drift is described by

$$
X(t)=\mu t+W(t),
$$

where $X(t)$ is the position of a realization of the stochastic process at a time $t, \mu$ is a positive constant which represents a drift velocity and $W(t)$ is a Brownian motion with the initial condition $W(t=0)=0$. A stochastic property of the Brownian motion is given by a normal distribution whose expected value and variance are,

$$
\langle W(t)\rangle=0, \quad\left\langle W^{2}(t)\right\rangle=t .
$$

Note that the Brownian motion can be represented as a time integrated white noise. Indeed, for the white noise $\xi(t)$ normalized as eq. (2.18), it can be checked easily that the Brownian motion $W(t)$ defined as $W(t)=\int_{0}^{t} \xi\left(t^{\prime}\right) d t^{\prime}$ satisfies eq. (3.2).

It is known that considering following quantity is useful to see characteristics of a stochastic process,

$$
Z(t)=\exp \left[\sigma X(t)-\left(\sigma \mu+\frac{1}{2} \sigma^{2}\right) t\right],
$$

where $\sigma$ is an arbitrary positive costant. This quantity is called exponential martingale. Provided that $X(t)$ is given by eq. (3.1) and its initial condition is $X(0)=0$, the expected value of $Z(t)$ can be computed as

$$
\begin{aligned}
\langle Z(t)\rangle & =\left\langle\exp \left(\sigma W(t)-\frac{1}{2} \sigma^{2} t\right)\right\rangle \\
& =\exp \left(-\frac{1}{2} \sigma^{2} t\right) \frac{1}{\sqrt{2 \pi t}} \int_{-\infty}^{\infty} d x \exp \left(\sigma x-\frac{x^{2}}{2 t}\right) \\
& =1 .
\end{aligned}
$$

where we have used the fact that the probability distribution of $W(t)$ is the normal distribution eq. (3.2). Therefore, if $X(0)=0$, the expectation value of $Z(t)$ is always 1 irrespective of $t$.

Next, we introduce the first passage time $\tau_{m} . \tau_{m}$ is defined as a time when a given realization of a stochastic process reaches $X(t)=m$ for its first time. Note that it is not an universal time but defined for each realization (called sample path) and it takes a different value for each sample path. In terms of inflation, it is nothing but the e-folding number $N$ because $N$ is the time which each separate universe takes from some initial flat slice to the end of inflation. Therefore the stochastic property of $\tau_{m}$ is crucial for the $\delta N$ formalism. To derive it, we substitute $t=\tau_{m}$ into eq. $(3.4)^{4}$ and obtain

$$
\left\langle\exp \left[\sigma m-\left(\sigma \mu+\frac{1}{2} \sigma^{2}\right) \tau_{m}\right]\right\rangle=1 .
$$

\footnotetext{
${ }^{4}$ It should be noted that $\tau_{m}$ depends on each sample path while $t$ is independent of paths and an universal time. Therefore the validity of this substitution looks non-trivial. However Doob's optional sampling theorem [30] guarantees its validity. One can also show that the probability of $\tau_{m} \rightarrow \infty$ converges to 0 [31].
} 
Defining a dummy parameter $J=\sigma \mu+\frac{1}{2} \sigma^{2}$, it reads

$$
\left\langle e^{-J \tau_{m}}\right\rangle=e^{m \mu-m \sqrt{2 J+\mu^{2}}} .
$$

It is a generating function of $\left\langle\tau_{m}^{n}\right\rangle$. By differentiating it $n$ times with respective to $J$ and taking $J \rightarrow 0$, one can obtain,

$$
\left\langle\tau_{m}\right\rangle=\frac{m}{\mu}, \quad\left\langle\tau_{m}^{2}\right\rangle=\frac{m^{2}}{\mu^{2}}+\frac{m}{\mu^{3}}, \quad\left\langle\tau_{m}^{3}\right\rangle=\frac{m^{3}}{\mu^{3}}+3 \frac{m^{2}}{\mu^{4}}+3 \frac{m}{\mu^{5}} .
$$

Note that $\left\langle\tau_{m}\right\rangle$ coincides with the result without the noise term $(\dot{X}=\mu)$. Furthermore we can also calculate, $\left\langle\delta \tau_{m}^{n}\right\rangle$, an expectation value of a $\mathrm{n}$-th power fluctuation of $\tau_{m}$,

$$
\left\langle\delta \tau_{m}\right\rangle=0, \quad\left\langle\delta \tau_{m}^{2}\right\rangle=\frac{m}{\mu^{3}}=\frac{1}{\mu^{2}}\left\langle\tau_{m}\right\rangle, \quad\left\langle\delta \tau_{m}^{3}\right\rangle=3 \frac{m}{\mu^{5}}=\frac{3}{\mu^{4}}\left\langle\tau_{m}\right\rangle,
$$

where $\delta \tau_{m}=\tau_{m}-\left\langle\tau_{m}\right\rangle$. Calculations for higher order perturbations are straightforward.

\subsection{Power spectrum}

In this subsection, we confirm the correspondence between the dynamics of a slow-rolling inflaton and the Brownian motion with the drift. Then we show the $n$-point correlation functions of the curvature perturbations can be obtained by connecting the $\delta N$ formalism with the result of the previous subsection. First of all, we assume that the Hubble parameter during inflation can be approximated by a linear function of $\phi$ as

$$
H(\phi) \simeq H_{0}-\alpha \phi,
$$

where $\alpha$ is supposed to be a positive constant for clarity. From eqs. (3.9) and (2.19), we obtain the equation of motion in the stochastic formalism,

$$
\dot{\phi}(t) \simeq 2 M_{p}^{2} \alpha+\frac{H_{0}^{3 / 2}}{2 \pi} \xi(t)
$$

where $\xi(t)$ is white noise whose variance is 1 ,

$$
\langle\xi(t)\rangle=0, \quad\left\langle\xi(t) \xi\left(t^{\prime}\right)\right\rangle=\delta\left(t-t^{\prime}\right) .
$$

Here, we regard the Hubble parameter as a constant by neglecting $-\alpha \phi$ in $H(\phi)$, except only for $-2 M_{p}^{2} H^{\prime}$. This makes the coefficient of the noise term constant and hence the coupling between the noise and the inflaton field is neglected. As we will see later, the power spectrum calculated in this subsection correspond with that of the standard linear perturbation theory exactly in these approximations.

Using $N=H_{0} t$, eq. (3.10) is rewritten as

$$
\frac{2 \pi}{H_{0}} \frac{d \phi}{d N}(N) \simeq \frac{4 \pi M_{p}^{2} \alpha}{H_{0}^{2}}+\tilde{\xi}(N),
$$

$\tilde{\xi}(N)$ is also normalized white noise $\left(\tilde{\xi}(N)=\xi(t) / H_{0}^{1 / 2}\right)$. If we define

$$
X(N)=\frac{2 \pi \phi}{H_{0}}, \quad \mu=\frac{4 \pi M_{p}^{2} \alpha}{H_{0}^{2}},
$$


the correspondence between the inflaton dynamics eq. (3.12) and the Brownian motion with the drift eq. (3.1) becomes transparent because integrated white noise is a Brownian motion as mentioned in subsection 3.1. In brief, when eq. (3.12) is integrated by $N$, it reads

$$
X(N)-X_{i}=\mu N+W(N)
$$

with $X_{i}$ denoting the initial value of $X(N)$.

Let us assume that slow-roll inflation ends when $\phi$ reaches $\phi_{f}$. Then, according to the $\delta N$ formalism, the fluctuation of the e-folding number $N$ defined between an initial flat slice and the $\phi=\phi_{f}$ slice is nothing but the curvature perturbations in real space. Here, since $N$ in the stochastic dynamics corresponds to the first passage time $\tau_{m}$ of the Brownian motion with the drift, one can find that the correlators of $\delta N$ can be computed in the exactly same way as $\left\langle\delta \tau_{m}^{n}\right\rangle$. Then we write the generating function,

$$
\left\langle e^{-J N}\right\rangle=e^{\left(X_{f}-X_{i}\right)\left(\mu-\sqrt{2 J+\mu^{2}}\right)},
$$

where $X_{f}$ is the final value of $X=2 \pi \phi / H_{0}$. Therefore we can calculate $\left\langle\delta N^{2}\right\rangle$ similarly to eq. (3.8) as

$$
\left\langle\delta N^{2}\right\rangle=\left\langle N^{2}\right\rangle-\langle N\rangle^{2}=\frac{1}{\mu^{2}}\langle N\rangle .
$$

On the other hand, a power spectrum of $\delta N$ is defined by a 2-point correlator of $N$,

$$
\mathcal{P}_{\delta N}(k)=\frac{k^{3}}{2 \pi^{2}} \int d^{3} x\langle\delta N(0) \delta N(x)\rangle e^{-i \mathbf{k} \cdot \mathbf{x}} .
$$

Provided that only modes which exits the horizon during inflation contribute to the variance of $N$, one can show

$$
\left\langle\delta N^{2}\right\rangle=\int_{k_{i}}^{k_{f}} \mathcal{P}_{\delta N}\left(k^{\prime}\right) \frac{d k^{\prime}}{k^{\prime}}=\int_{\ln k_{f}-\langle N\rangle}^{\ln k_{f}} \mathcal{P}_{\delta N} d N .
$$

Here, $k_{f}=a_{f} H_{0}, k_{i}=a_{i} H_{0}=k_{f} e^{-\langle N\rangle}$ and subscript $i$ and $f$ represent some initial slice and the end of inflation respectively. Therefore we obtain

$$
\mathcal{P}_{\zeta}(k)=\mathcal{P}_{\delta N}(k)=\left.\frac{d}{d\langle N\rangle}\left\langle\delta N^{2}\right\rangle\right|_{\langle N\rangle=\ln \left(k_{f} / k\right)}=\frac{1}{\mu^{2}}=\left(\frac{H_{0}^{2}}{4 \pi M_{p}^{2} \alpha}\right)^{2} .
$$

Using a slow-roll parameter $\epsilon_{H}=2 M_{p}^{2}\left(H^{\prime} / H\right)^{2} \simeq 2 M_{p}^{2} \alpha^{2} / H_{0}^{2}$ and the Friedmann equation $V \simeq 3 M_{p}^{2} H_{0}^{2}$, we obtain the exactly same result as the standard linear perturbation theory,

$$
\mathcal{P}_{\zeta}=\frac{1}{24 \pi^{2} M_{p}^{4}} \frac{V}{\epsilon_{H}}
$$

It is the main result of this paper.

What does our main result mean? Even in more general cases where the Hubble parameter can not be approximated linearly, as long as inflation is a single field slow-roll type, we can obtain the same result by dividing the whole inflation periods into shorter periods during which the linear approximation of the Hubble makes sense. In this case, the right 
hand side of eq. (3.20) will be evaluated at the horizon exit of the scale of the interest because the Hubble parameter $H$ and its gradient $\alpha$ vary over each period. That is because the super-horizon scale curvature perturbations are conserved in single field inflation models. Therefore, we do not have to wait until the end of inflation and can take the final slice of the $\delta N$ formalism a certain time when the scale of the interest becomes sufficiently larger than the horizon. Of course, in order to use the $\delta N$ formalism, it is needed that the end of the period can be taken well after the horizon exit of the interest mode, However as long as the slow-roll approximation is good enough (i.e. the Hubble parameter does not vary rapidly), it seems possible to take such a period. Therefore it is suggested that the power spectrum of the curvature perturbations will correspond with the standard result even if the stochastic effect is considered in single slow-roll inflation.

It should be noted that in our formalism, the condition that the quantum kicks are smaller than the classical motion is not required at all because we do not expand the inflaton field perturbatively. In general, it is said that inflation is stochastic if the quantum kicks are comparable to or bigger than the classical motion. Since $\mu$ in eq. (3.13) represents the ratio of the classical motion to the quantum kicks, inflation is stochastic for $\mu^{-1}\left(=\mathcal{P}_{\zeta}^{1 / 2}\right) \gtrsim 1 .^{5}$ Apparently it seems that the larger the power spectrum became (i.e. the more stochastic inflation becomes), the worse the linear perturbation theory is. However our main result implies that even if inflation is stochastic to a certain extent, the linear perturbation theory gives a precise result for single field slow-roll inflation. It is because in single field slowroll inflation the non-Gaussianity of the curvature perturbations is small even if the power spectrum is large. In the $\delta N$ formalism, when the non-Gaussianity is small, the first order approximation,

$$
\zeta=\delta N \simeq \frac{\partial N}{\partial \phi} \delta \phi
$$

is good approximation regardless of the amplitude of $\delta N$ and it is nothing but the linear perturbation theory. Our result not only verifies our formalism but also guarantees that the linear perturbation theory gives the precise curvature perturbations in single field slow-roll inflation even if the quantum fluctuations are comparable to classical dynamics.

\subsection{Non-Gaussianity}

As we see in section 3.1, we can calculate higher order expected values of the e-foldings easily in our formalism. Therefore we can also obtain the non-Gaussianity of the curvature perturbations. One can expect that the curvature perturbations are almost Gaussian because we consider single field slow-roll inflation models. Let us check it. For this purpose, the quantities such as the skewness which vanishes in an exact Gaussian case is useful. The skewness $S_{\zeta}$ is defined by $S_{\zeta}:=\left\langle\zeta^{3}\right\rangle /\left\langle\zeta^{2}\right\rangle^{3 / 2}$ and in our case it is given by,

$$
S_{\zeta}=\frac{\left\langle\zeta^{3}\right\rangle}{\left\langle\zeta^{2}\right\rangle^{3 / 2}}=\frac{3}{\mu} \frac{1}{\langle N\rangle^{1 / 2}}=\mathcal{P}_{\zeta}^{1 / 2} \frac{3}{\langle N\rangle^{1 / 2}}
$$

Here we have used the result of section 3.1 for $\left\langle\zeta^{3}\right\rangle$. In the observational aspects, the super-horizon modes for the current observable universe can not be distinguished from the

\footnotetext{
${ }^{5}$ Since $\mathcal{P}_{\zeta}$ should be $\lesssim 10^{-2}$ due to the PBH constraints [39], we do not have to consider very large curvature perturbations.
} 
homogeneous zero mode. We can observe only the sub-horizon modes corresponding to $k_{f}>k>k_{f} e^{-N_{\text {obs }}}$ where $N_{\text {obs }} \sim 60$. Therefore the expected values should be averaged over the current observable universe, and then we take $\langle N\rangle \sim 60$. If the power spectrum is consistent with the observed value $\mathcal{P}_{\zeta}^{1 / 2} \sim 10^{-5}$, the skewness is indeed much less than unity and then curvature perturbations are indeed almost Gaussian.

The commonly used nonlinearity parameter $f_{\mathrm{NL}}$ is also useful. Let us calculate the local type nonlinearity parameter by approximating curvature perturbations as

$$
\zeta(\mathbf{x}) \simeq g(\mathbf{x})+\frac{3}{5} f_{\mathrm{NL}}^{\text {loc }}\left(g^{2}(\mathbf{x})-\left\langle g^{2}(\mathbf{x})\right\rangle\right),
$$

where $g(\mathbf{x})$ is a Gaussian fluctuation. Since curvature perturbations are almost Gaussian, $f_{\mathrm{NL}}^{\text {loc }}$ is expected to be much less than unity. Now the squared and cubic expected values are

$$
\begin{aligned}
& \left\langle\zeta^{2}\right\rangle=\left\langle g^{2}\right\rangle+\mathcal{O}\left(f_{\mathrm{NL}}^{\mathrm{loc} 2}\right), \\
& \left\langle\zeta^{3}\right\rangle=\frac{9}{5} f_{\mathrm{NL}}^{\mathrm{loc}}\left(\left\langle g^{4}\right\rangle-\left\langle g^{2}\right\rangle^{2}\right)+\mathcal{O}\left(f_{\mathrm{NL}}^{\mathrm{loc} 3}\right) \simeq \frac{18}{5} f_{\mathrm{NL}}^{\mathrm{loc}}\left\langle g^{2}\right\rangle^{2},
\end{aligned}
$$

where we used the Wick's theorem $\left\langle g^{4}\right\rangle=3\left\langle g^{2}\right\rangle^{2}$. Therefore at leading order we obtain

$$
f_{\mathrm{NL}}^{\mathrm{loc}} \simeq \frac{5}{18} \frac{\left\langle\zeta^{3}\right\rangle}{\left\langle\zeta^{2}\right\rangle^{2}}=\frac{5}{6} \frac{1}{\langle N\rangle} \sim \frac{1}{72}
$$

For the same reason we mentioned above, we take $\langle N\rangle \sim 60$.

For comparison with it, let us calculate the nonlinearity parameter by the standard linear perturbation theory, too. In the $\delta N$ formalism, taking the second order perturbations of the inflaton field,

$$
\zeta(\mathbf{x})=\delta N(\mathbf{x}) \simeq \frac{\partial N}{\partial \phi} \delta \phi(\mathbf{x})+\frac{1}{2} \frac{\partial^{2} N}{\partial \phi^{2}} \delta \phi^{2}(\mathbf{x})
$$

Because the inflaton is assumed to be almost massless, $\delta \phi$ is a nearly Gaussian fluctuation and we can assume $g(\mathbf{x})=N^{\prime} \delta \phi(\mathbf{x})$. Therefore, comparing with eq. (3.23), we obtain

$$
\frac{3}{5} f_{\mathrm{NL}}^{\text {loc }}=\frac{1}{2} \frac{N^{\prime \prime}}{\left(N^{\prime}\right)^{2}}
$$

The equation of motion without noise is

$$
H \frac{d \phi}{d N}=-2 M_{p}^{2} H^{\prime}
$$

By integrating it, one can find the e-foldings number and its derivatives,

$$
N=\frac{1}{2 M_{p}^{2} \alpha} \int_{\phi}^{\phi_{f}}\left(H_{0}-\alpha \phi^{\prime}\right) d \phi^{\prime}, \quad N^{\prime} \simeq-\frac{H_{0}}{2 M_{p}^{2} \alpha}, \quad N^{\prime \prime}=\frac{1}{2 M_{p}^{2}}
$$

Then the nonlinearity parameter is given by

$$
f_{\mathrm{NL}}^{\text {loc }}=\frac{5}{6} \times \frac{2 M_{p}^{2} \alpha^{2}}{H_{0}^{2}}=\frac{5}{6} \epsilon_{H} .
$$


Although it is apparently different from the stochastic result eq. (3.26), they are consistent actually. With the definition of the slow-roll parameter $\epsilon_{H}=2 M_{p}^{2}\left(H^{\prime} / H\right)^{2}$ and the slow-roll equation of motion $\dot{\phi}+2 M_{p}^{2} H^{\prime}=0$, we rewrite the slow-roll parameter

$$
\epsilon_{H}=-\frac{\dot{H}}{H^{2}}
$$

It can be deformed as

$$
H d t=-\frac{1}{\epsilon_{H}} \frac{d H}{H}
$$

Then supposing the time dependence of the slow-roll parameter can be neglected, it reads

$$
\int_{t_{i}}^{t_{f}} H d t=\frac{1}{\epsilon_{H}} \log \left(\frac{H_{i}}{H_{f}}\right) .
$$

The left hand side is nothing but the precise definition of the e-foldings $N$. In slow-roll inflation, $\log \left(H_{i} / H_{f}\right)$ is expected to be an order one factor, so it has been proved that the e-foldings $N$ and the inverse of the slow-roll parameter $\epsilon_{H}^{-1}$ have the same order of magnitude. As a result, the nonlinearity parameter in the stochastic formalism is consistent with that obtained in the standard linear perturbation theory.

\section{Extension to more general case}

Though we explain the analytic calculation of the power spectrum and the non-Gaussianity in single field slow-roll inflation in the last section, we have to solve the equation of motion numerically in general cases. In this section, we briefly describe the algorithm for numerical calculations. For single field inflation models,

1. Choose a certain initial value $\phi_{i}$ for the inflaton field $\phi$ temporarily.

2. Start inflation from that temporary initial value $\phi_{i}$, integrate the full equations of motion,

$$
\left\{\begin{array}{l}
H \frac{d \phi}{d N}=\pi+\frac{H^{2}}{2 \pi} \xi(N), \\
H \frac{d \pi}{d N}=-3 H \pi-\frac{d V}{d \phi}
\end{array}\right.
$$

numerically and calculate the e-foldings $N$ which is needed to reach the end value $\phi_{f}$ (for example, the point where the slow-roll parameter becomes unity). In the numerical integration of eqs. (4.1) and (4.2), the noise term $\xi(N)$ is replaced with a random variable obeying a normal distribution whose expected value and variance are 0 and $\Delta N$ where $\Delta N$ is the step of the numerical integration. Therefore, the calculated efolding varies in each calculation (realization). Repeating this calculation many times, we can get $\langle N\rangle,\left\langle\delta N^{2}\right\rangle,\left\langle\delta N^{3}\right\rangle$ and so on.

3. Reiterating the above process for various initial values, we obtain the relation between $\langle N\rangle$ and $\left\langle\delta N^{2}\right\rangle$ (or $\left\langle\delta N^{3}\right\rangle$ ), and thus $\left\langle\delta N^{2}\right\rangle$ and $\left\langle\delta N^{3}\right\rangle$ can be represented as functions of $\langle N\rangle$. 
4. Finally, differentiating $\left\langle\delta N^{2}\right\rangle$ by $\langle N\rangle$, we can obtain the power spectrum of curvature perturbations,

$$
\mathcal{P}_{\zeta}(k)=\left.\frac{d\left\langle\delta N^{2}\right\rangle}{d\langle N\rangle}\right|_{\langle N\rangle=\ln k_{f}-\ln k} .
$$

We can also obtain the local type nonlinearity parameter $f_{\mathrm{NL}}^{\text {loc }}$ when the curvature perturbations are almost Gaussian,

$$
\left.f_{\mathrm{NL}}^{\mathrm{loc}} \simeq \frac{\left\langle\delta N^{3}\right\rangle}{\left\langle\delta N^{2}\right\rangle^{2}}\right|_{\langle N\rangle \sim 60} .
$$

If the curvature perturbations are not Gaussian at all, one should use the full simultaneous equations,

$$
\left\{\begin{array}{l}
\left\langle\zeta^{2}\right\rangle=\left\langle g^{2}\right\rangle+\frac{18}{25} f_{\mathrm{NL}}^{\text {loc } 2}\left\langle g^{2}\right\rangle^{2} \\
\left\langle\zeta^{3}\right\rangle=\frac{18}{5} f_{\mathrm{NL}}^{\text {loc }}\left\langle g^{2}\right\rangle^{2}+\frac{216}{125} f_{\mathrm{NL}}^{\text {loc } 3}\left\langle g^{2}\right\rangle^{3}
\end{array}\right.
$$

for $f_{\mathrm{NL}}^{\text {loc }}$ and $\left\langle g^{2}\right\rangle$.

The extension to multi-field inflation models is also interesting. In a multi-field case, however, the choice of the temporary initial field values is not trivial. It is because the solution of the equation of motion with noise (called sample path) can not be determined uniquely because the field space is multi-dimensional and can be different from the no noise solution (called classical path). Thus a careful treatment is necessary. We propose the following procedure.

1. Choose some fixed initial values for relevant fields. These initial values must be placed at regions where the inflaton potential is high enough for inflation to last at least 60 e-foldings.

2. Produce a lot of sample path from that fixed initial value. Each sample path is assumed to have a same probabilistic weight. It is the assumption of stochastic calculus.

3. For each sample path, take various temporary initial values on the sample path and calculate the power spectrum similarly to single field inflation.

4. The true power spectrum should be identified with the mean of those obtained from many sample paths. In other words, since each sample path has same probabilistic weight, sum up the power spectrum calculated for each sample path and divide it by the number of sample paths.

In this algorithm, all sample paths are produced from one fixed initial values. It means that in the sufficiently early phase of inflation, the inflaton field values were same at least over the observable universe, and thereafter each horizon evolves separately. It is valid because the observable universe was in one same horizon more than 60 e-foldings before the end of inflation and the assumption that the inflaton field values were same at that time is natural. There is a problem concerning the fixed initial values. Generally, if there is more than one scalar fields whose masses are much lighter than the Hubble parameter, the paths followed 
by the inflaton fields quite depend on their initial values. Thus, different choices of the initial values may result in different power spectra. However, in many cases, such as the hybrid inflation model, the inflaton field value will converge to a classical attractor path soon without dependence on the initial values. Therefore, one can take the fixed initial values on that classical attractor in such cases.

\section{Conclusion}

In this paper, we propose a new algorithm for calculating the curvature perturbations produced during inflation in the stochastic formalism. In our algorithm, the curvature perturbations can be calculated non-perturbatively. As mentioned in section 3, in single field slow-roll inflation, we can obtain the curvature perturbations analytically in our formalism with the help of the techniques of stochastic calculus, and the calculated power spectrum and non-Gaussianity of the curvature perturbations are consistent with the standard result. It is quite natural because the curvature perturbations produced in single slow-roll inflation are almost Gaussian and the linear perturbation approach (i.e. the standard approach) works well. Therefore, the power spectrum is not expected to be so different from linear perturbation result even if the stochastic effect is considered. In more general cases, such as highly non-Gaussian cases, our formalism is expected to give more precise result than the standard linear approach. Therefore, we also described how the curvature perturbations can be calculated by our formalism in such cases in section 4 . Even though it is known that the non-Gaussianity is so small at CMB scale, it may not be negligible at smaller scales. Furthermore, the power spectrum at small scales may also be large and some interesting objects like PBHs and UCMHs can be formed. Our non-perturbative formalism is useful for studying such cases.

\section{Acknowledgments}

We would like to thank Shuichiro Yokoyama for helpful discussions. This work is supported by Grant-in-Aid for Scientific research from the Ministry of Education, Science, Sports, and Culture (MEXT), Japan, No. 25400248 [MK], No. 21111006 [MK] and also by World Premier International Research Center Initiative (WPI Initiative), MEXT, Japan. T.F. and T.T. acknowledge the support by JSPS Research Fellowships for Young Scientists. The work of Y.T. is partially supported by an Advanced Leading Graduate Course for Photon Science grant.

\section{References}

[1] A. H. Guth, "The Inflationary Universe: A Possible Solution to the Horizon and Flatness Problems," Phys. Rev. D 23, 347 (1981).

[2] A. A. Starobinsky, "A New Type of Isotropic Cosmological Models Without Singularity," Phys. Lett. B 91, 99 (1980).

[3] K. Sato, "First Order Phase Transition of a Vacuum and Expansion of the Universe," Mon. Not. Roy. Astron. Soc. 195, 467 (1981).

[4] A. D. Linde, "A New Inflationary Universe Scenario: A Possible Solution of the Horizon, Flatness, Homogeneity, Isotropy and Primordial Monopole Problems," Phys. Lett. B 108, 389 (1982). 
[5] A. Albrecht and P. J. Steinhardt, "Cosmology for Grand Unified Theories with Radiatively Induced Symmetry Breaking," Phys. Rev. Lett. 48, 1220 (1982).

[6] R. Brout, F. Englert and E. Gunzig, "The Creation of the Universe as a Quantum Phenomenon," Annals Phys. 115, 78 (1978).

[7] A. A. Starobinsky, "Relict Gravitation Radiation Spectrum and Initial State of the Universe. (In Russian)," JETP Lett. 30, 682 (1979) [Pisma Zh. Eksp. Teor. Fiz. 30, 719 (1979)].

[8] D. Kazanas, "Dynamics of the Universe and Spontaneous Symmetry Breaking," Astrophys. J. 241, L59 (1980).

[9] P. A. R. Ade et al. [Planck Collaboration], "Planck 2013 results. I. Overview of products and scientific results," arXiv:1303.5062 [astro-ph.CO].

[10] A. Vilenkin, "The Birth of Inflationary Universes," Phys. Rev. D 27, 2848 (1983).

[11] A. D. Linde, "Eternally Existing Selfreproducing Chaotic Inflationary Universe," Phys. Lett. B 175, 395 (1986).

[12] A. A. Starobinsky, "Stochastic De Sitter (inflationary) Stage In The Early Universe," In *De Vega, H.j. ( Ed.), Sanchez, N. ( Ed.): Field Theory, Quantum Gravity and Strings*, 107-126 (1986).

[13] A. A. Starobinsky and J. Yokoyama, "Equilibrium state of a selfinteracting scalar field in the De Sitter background," Phys. Rev. D 50, 6357 (1994) [astro-ph/9407016].

[14] M. Sasaki, Y. Nambu and K. -i. Nakao, "Classical Behavior Of A Scalar Field In The Inflationary Universe," Nucl. Phys. B 308, 868 (1988).

[15] K. -i. Nakao, Y. Nambu and M. Sasaki, "Stochastic Dynamics Of New Inflation," Prog. Theor. Phys. 80, 1041 (1988).

[16] Y. Nambu and M. Sasaki, "Stochastic Approach To Chaotic Inflation And The Distribution Of Universes," Phys. Lett. B 219, 240 (1989).

[17] S. Mollerach, S. Matarrese, A. Ortolan and F. Lucchin, "Stochastic inflation in a simple two field model," Phys. Rev. D 44, 1670 (1991).

[18] J. Martin and V. Vennin, "Stochastic Effects in Hybrid Inflation," Phys. Rev. D 85, 043525 (2012) [arXiv:1110.2070 [astro-ph.CO]].

[19] K. Enqvist, D. G. Figueroa and G. Rigopoulos, "Fluctuations along supersymmetric flat directions during Inflation," JCAP 1201, 053 (2012) [arXiv:1109.3024 [astro-ph.CO]].

[20] M. Kawasaki and T. Takesako, "Stochastic Approach to Flat Direction during Inflation," JCAP 1208, 031 (2012).

[21] F. Finelli, G. Marozzi, A. A. Starobinsky, G. P. Vacca and G. Venturi, "Generation of fluctuations during inflation: Comparison of stochastic and field-theoretic approaches," Phys. Rev. D 79, 044007 (2009) [arXiv:0808.1786 [hep-th]].

[22] F. Finelli, G. Marozzi, A. A. Starobinsky, G. P. Vacca and G. Venturi, "Stochastic growth of quantum fluctuations during slow-roll inflation," Phys. Rev. D 82, 064020 (2010) [arXiv:1003.1327 [hep-th]].

[23] J. C. B. Sanchez and K. Enqvist, "On the fate of coupled flat directions during inflation," JCAP 1303 (2013) 029 [arXiv:1210.7007 [astro-ph.CO]].

[24] J. C. B. Sanchez and K. Dimopoulos, "Inflationary buildup of a vector field condensate and its cosmological consequences," arXiv:1308.3739 [hep-ph].

[25] D. H. Lyth, K. A. Malik and M. Sasaki, "A General proof of the conservation of the curvature perturbation," JCAP 0505, 004 (2005) [astro-ph/0411220]. 
[26] A. A. Starobinsky, "Multicomponent de Sitter (Inflationary) Stages and the Generation of Perturbations," JETP Lett. 42, 152 (1985) [Pisma Zh. Eksp. Teor. Fiz. 42, 124 (1985)].

[27] D. S. Salopek and J. R. Bond, "Nonlinear evolution of long wavelength metric fluctuations in inflationary models," Phys. Rev. D 42, 3936 (1990).

[28] M. Sasaki and E. D. Stewart, "A General analytic formula for the spectral index of the density perturbations produced during inflation," Prog. Theor. Phys. 95, 71 (1996) [astro-ph/9507001].

[29] M. Sasaki and T. Tanaka, "Superhorizon scale dynamics of multiscalar inflation," Prog. Theor. Phys. 99, 763 (1998) [gr-qc/9801017].

[30] J. L. Doob, "Stochastic Processes," J. Wiley and Sons, New York. (1953).

[31] S. E. Shreve, "Stochastic Calculus for Finance II: Continuous-Time Models," Springer-Verlog New York, LLC. (2004).

[32] K. E. Kunze, "Perturbations in stochastic inflation," JCAP 0607, 014 (2006) [astro-ph/0603575].

[33] J. Martin and M. Musso, "Solving stochastic inflation for arbitrary potentials," Phys. Rev. D 73, 043516 (2006) [hep-th/0511214].

[34] J. Martin and M. Musso, "On the reliability of the Langevin perturbative solution in stochastic inflation," Phys. Rev. D 73, 043517 (2006) [hep-th/0511292].

[35] L. Perreault Levasseur, "Lagrangian Formulation of Stochastic Inflation: A Recursive Approach," arXiv:1304.6408 [hep-th].

[36] L. Perreault Levasseur, V. Vennin and R. Brandenberger, "Recursive Stochastic Effects in Valley Hybrid Inflation," arXiv:1307.2575 [hep-th].

[37] S. Winitzki and A. Vilenkin, "Effective noise in stochastic description of inflation," Phys. Rev. D 61, 084008 (2000) [gr-qc/9911029].

[38] H. Casini, R. Montemayor and P. Sisterna, "Stochastic approach to inflation. 2. Classicality, coarse graining and noises," Phys. Rev. D 59, 063512 (1999) [gr-qc/9811083].

[39] B. J. Carr, K. Kohri, Y. Sendouda and J. 'i. Yokoyama, "New cosmological constraints on primordial black holes," Phys. Rev. D 81, 104019 (2010) [arXiv:0912.5297 [astro-ph.CO]].

[40] B. J. Carr, "The Primordial black hole mass spectrum," Astrophys. J. 201, 1 (1975).

[41] B. J. Carr, "Some cosmological consequences of primordial black-hole evaporations," Astrophys. J. 206, 8 (1976).

[42] B. J. Carr, "Primordial black holes as a probe of cosmology and high energy physics," Lect. Notes Phys. 631, 301 (2003) [astro-ph/0310838].

[43] T. Bringmann, P. Scott and Y. Akrami, "Improved constraints on the primordial power spectrum at small scales from ultracompact minihalos," Phys. Rev. D 85, 125027 (2012) [arXiv:1110.2484 [astro-ph.CO]].

[44] A. S. Josan and A. M. Green, "Gamma-rays from ultracompact minihalos: potential constraints on the primordial curvature perturbation," Phys. Rev. D 82, 083527 (2010) [arXiv:1006.4970 [astro-ph.CO]].

[45] F. Li, A. L. Erickcek and N. M. Law, "A new probe of the small-scale primordial power spectrum: astrometric microlensing by ultracompact minihalos," Phys. Rev. D 86, 043519 (2012) [arXiv:1202.1284 [astro-ph.CO]]. 